\title{
RESEARCH
}

Open Access

\section{Effects of yoga on exercise capacity in patients with lymphangioleiomyomatosis: a nonrandomized controlled study}

Xiangfeng $\mathrm{Li}^{1+}$, Wenshuai $\mathrm{Xu}^{2+}{ }^{+}$, Lu Zhang ${ }^{3+}, \mathrm{Yi}_{\mathrm{Zu}}{ }^{4}, \mathrm{Yu} \mathrm{Li}^{5+}$, Yanli Yang${ }^{2}$, Ying Xiang ${ }^{4}$, Yun Xiang ${ }^{4}$, Ling Chen ${ }^{5}$, Wei Liu ${ }^{6}$, Lixia Chen ${ }^{3}$ and Kai-Feng $X^{2,7^{*}}$ (D)

\begin{abstract}
Objective: To evaluate the effects of yoga on exercise capacity and quality of life in patients with lymphangioleiomyomatosis (LAM), a rare cystic lung disease in women.

Patients and methods: This was a nonrandomized, controlled study conducted in Beijing, China (August 27, 2017 - April 26, 2018). Twenty-six participants were allocated to the intervention (yoga) group $(n=13)$ or control group $(n=13)$. The yoga intervention involved a 24-week program of yoga class training for 90 min once a week and no fewer than 2 at-home sessions per week (at least 15 min per session). The 6-min walking distance (6MWD), lung function, serum vascular endothelial growth factor-D (VEGF-D) levels, quality of life, and symptoms of anxiety and depression were measured at baseline, 12-week and 24-week follow-up. An incremental cardiopulmonary exercise test was conducted at baseline and the 24-week follow-up.
\end{abstract}

Results: Eleven patients completed the yoga training program. The yoga group exhibited improvements in the following outcomes versus those of the control group: 6MWD ( $+55 \pm 29 \mathrm{~m}$ vs $+18 \pm 49 \mathrm{~m}, P=0.04)$, anaerobic threshold $(3.4 \pm 2.4 \mathrm{ml} / \mathrm{min} / \mathrm{kg}$ vs $1.6 \pm 1.4 \mathrm{ml} / \mathrm{min} / \mathrm{kg}, P=0.035)$ and peak work load $(11.7 \pm 14.6 \mathrm{~W}$ vs $0.2 \pm 9.1 \mathrm{~W}$, $P=0.027)$. There was no significant difference in peak oxygen consumption $\left(\mathrm{VO}_{2}\right.$ peak), lung function, VEGF-D level, and quality of life between the yoga and control groups. No adverse effects were found in the yoga group.

Conclusion: Yoga is a feasible and safe intervention for pulmonary rehabilitation and potentially improves exercise capacity in patients with LAM.

Trial registration: (Clinical trial registration number at www.chictr.org.cn: ChiCTR-OON-1701274)

Keywords: Lymphangioleiomyomatosis, Pulmonary rehabilitation, Yoga, Exercise capacity

\footnotetext{
* Correspondence: xukf@pumch.cn

${ }^{+}$Xiangfeng Li, Wenshuai Xu, Lu Zhang and Yu Li contributed equally to this work.

${ }^{2}$ Department of Pulmonary and Critical Care Medicine, Peking Union Medical College Hospital, Chinese Academy of Medical Sciences, Peking Union Medical College, Beijing 100730, China

${ }^{7}$ Rare Diseases Research Center, Chinese Academy of Medical Sciences, Beijing, China

Full list of author information is available at the end of the article
}

\section{Background}

Lymphangioleiomyomatosis (LAM) is a rare disease that typically occurs in females and is characterized as diffuse pulmonary cystic changes [1]. The symptoms include progressive dyspnea, hypoxia, recurrent pneumothorax and chylothorax. LAM can be effectively treated with sirolimus (rapamycin), which slows disease progression [2]. Most LAM patients complain of reduced exercise capacity and impaired quality of life because of airflow obstruction, abnormal diffusion capacity, dynamic

(c) The Author(s). 2020 Open Access This article is licensed under a Creative Commons Attribution 4.0 International License, which permits use, sharing, adaptation, distribution and reproduction in any medium or format, as long as you give appropriate credit to the original author(s) and the source, provide a link to the Creative Commons licence, and indicate if changes were made. The images or other third party material in this article are included in the article's Creative Commons licence, unless indicated otherwise in a credit line to the material. If material is not included in the article's Creative Commons licence and your intended use is not permitted by statutory regulation or exceeds the permitted use, you will need to obtain permission directly from the copyright holder. To view a copy of this licence, visit http://creativecommons.org/licenses/by/4.0/. The Creative Commons Public Domain Dedication waiver (http://creativecommons.org/publicdomain/zero/1.0/) applies to the data made available in this article, unless otherwise stated in a credit line to the data. 
hyperinflation, peripheral muscle dysfunction or pulmonary hypertension [3].

Pulmonary rehabilitation consisting of a 24-week aerobic exercise and muscle strength training significantly improved endurance time, St. George Respiratory Questionnaire (SGRQ) scores, 6-min walking distance (6MWD), dyspnea and peak oxygen consumption ( $\mathrm{VO}_{2}$ peak) [4]. Yoga is an attractive alternative option of pulmonary rehabilitation for LAM patients. The current evidence suggests that yoga exercises have beneficial effects on improvements in lung function and exercise capacity and could be used as an adjunct pulmonary rehabilitation program in patients with chronic obstructive pulmonary disease (COPD) [5]. Exertional dyspnea is an important burden associated with LAM, and many of the mechanisms are like those described in COPD. Therefore, we hypothesized that LAM patients will benefit from yoga training. In the present study, we aimed to assess the effects and safety of yoga on cardiopulmonary exercise tests, lung function, quality of life, dyspnea, anxiety and depression in LAM patients.

\section{Methods}

\section{Study design and participants}

The investigation was a nonrandomized, controlled, parallel study conducted in Beijing, China. All LAM patients registered in the National Rare Diseases Registry System of China (NRDRS) were evaluated for study participation. Patients had to meet the following criteria: age not less than 18 years old; living in Beijing city (for the yoga group); diagnosis of LAM according to the American Thoracic Society and Japanese Respiratory Society guidelines [6]; and clinical stability defined as no exacerbations for a minimum of 8 weeks and no change in treatment in the previous 8 weeks.

Patients were excluded for any of the following conditions: after lung transplantation or currently waiting on a lung transplantation list; pregnancy or lactation; musculoskeletal or cognitive disorders that could interfere with testing; unstable angina, respiratory tract infection within 1 month of the start of the study; previous involvement in yoga rehabilitation programs or other pulmonary rehabilitation programs within 3 months; and history of pneumothorax during the past 3 months. The protocol was approved by the Peking Union Medical College Hospital ethical committee (ZS-1398), and all patients provided written informed consent before enrolment. The study was registered at the Chinese Clinical Trial Registry (http://www.chictr.org.cn) and was assigned the following identifier: ChiCTR-OON-17012748.

The decision to perform a nonrandomized trial was made due to the rarity of the disease and geographical issues. Prior to the study, we stated that patients from Beijing would be invited to participate in the intervention, whereas patients living outside Beijing or unable to attend the yoga exercise sessions for other reasons would be invited as controls.

\section{Yoga intervention}

Traditional hatha yoga was used in this project. The yoga intervention was carried out in a well-ventilated yoga classroom and included yoga class training once a week (90 min for each session) and home exercise of yoga that they were trained in class no fewer than 2 times a week (at least 15 min per time) during 24 weeks (from August 2017 to April 2018). The intervention was provided by certified yoga instructors who were not involved in the measurements. The curriculum was specifically designed for LAM patients based on the baseline measurement and individual physical condition. The patients were offered yoga sessions that consisted of yoga asanas (poses) interspersed with chanting and pranayama (timed breathing). The exercise was performed in a sequential, systematic and scientific way aiming at improving the overall status of the participants. The yoga participants were supposed to be able to awaken the body, know the body, and strengthen the body.

\section{Measurements}

The primary outcome was walking distance during the 6min walk test (6MWD). 6-min -walk test of all the patients were performed in Peking Union Medical College Hospital [7]. The secondary outcomes included $\mathrm{VO}_{2}$ peak, anaerobic threshold (AT) during the incremental cardiopulmonary exercise test, forced expiratory volume at $1 \mathrm{~s}\left(\mathrm{FEV}_{1}\right)$ and forced vital capacity (FVC) measured using spirometry, daily physical activity assessed using a sports bracelet (Mambo 2, manufactured by Lifesense Medical Electronics Co., Ltd., Guangdong, China); dyspnea assessed by the modified Borg scale on a score of $0 \sim 10$ on cessation of the 6-min walk test; health-related quality of life using the St. George Respiratory Questionnaire (SGRQ); symptoms of anxiety and depression using the Hospital Anxiety and Depression Scale (HADS). Safety was assessed by the occurrence of adverse events, including pneumothorax and other conditions. Adherence was assessed by the percentage of yoga sessions attended and the number of exercise sessions at home (self-reported diary).

\section{Statistical issues}

Data were analyzed using SPSS for Windows version 20.0 (IBM Corp., USA) and are reported as the mean \pm $\mathrm{SD}$. The unpaired $t$-test or Mann-Whitney $U$-test was used to compare continuous variables. Categorical variables were compared using Fisher's exact test. The paired $t$-test was used to compare within-subject results. 
For all analyses, two-sided tests and a significance level of 0.05 was used. Intention-to-treat analyses were used for data analysis.

\section{Results}

Of the LAM patients registered in the NRDRS, 13 patients each were assigned to the yoga and control groups (August 27, 2017 - April 26, 2018). Both groups were well balanced in clinical characteristics, lung function and exercise capacity (Table 1). One patient in the yoga group did not complete the program (absent from 13 sessions), and another patient in the yoga group presented increased chylous ascites. Eleven patients in the yoga group and 13 patients in the control group completed the program at 24 weeks (Fig. 1). The median attendance in the yoga group was $91.7 \%$ (IQR, 87.5, 100\%). Patients did not attend the classes for reasons involving physical complaints (menstrual periods or colds) or time conflicts because of their jobs. The median home exercise duration per week was $75 \mathrm{~min}$ (IQR, 72.4, $104.3 \mathrm{~min}$ ).

Table 1 Baseline characteristics of the participants in the two groups

\begin{tabular}{|c|c|c|c|}
\hline Characteristics & Yoga $(\boldsymbol{N}=13)$ & Control $(\mathrm{N}=13)$ & $\boldsymbol{P}$ Value \\
\hline Age-years & $39.8 \pm 8.5$ & $43.4 \pm 9.2$ & 0.317 \\
\hline BMI $\left(\mathrm{kg} / \mathrm{m}^{2}\right)$ & $21.9 \pm 2.2$ & $20.9 \pm 1.5$ & 0.201 \\
\hline Daily steps & $9383 \pm 3885$ & $9388 \pm 3962$ & 0.998 \\
\hline $6 \mathrm{MWD}(\mathrm{m})$ & $550 \pm 64$ & $516 \pm 80$ & $0.209 t$ \\
\hline $\mathrm{SpO}_{2}$ after exercise (\%) & $88 \pm 9$ & $90 \pm 8$ & 0.507 \\
\hline Borg score after exercise & $0.9 \pm 1.3$ & $1.1 \pm 1.4$ & $0.978 t$ \\
\hline VEGF-D, pg/ml & $2957 \pm 2915$ & $2785 \pm 3024$ & $0.369 t$ \\
\hline \multicolumn{4}{|l|}{ Clinical features - no. (\%) } \\
\hline Sirolimus treatment & $6(46)$ & $7(54)$ & 1.0009 \\
\hline History of pneumothorax & $6(46)$ & $3(23)$ & 0.4119 \\
\hline History of angiomyolipoma & $1(8)$ & $5(38)$ & 0.1609 \\
\hline Tuberous sclerosis complex & $1(8)$ & $1(8)$ & 1.0009 \\
\hline History of chylothorax or ascites & $5(38)$ & $3(23)$ & 0.6739 \\
\hline \multicolumn{4}{|c|}{ St. George Respiratory Questionnaire (SGRQ) } \\
\hline Symptoms & $16.0 \pm 13.1$ & $15.0 \pm 17.0$ & $0.537 t$ \\
\hline Activity & $30.1 \pm 14.7$ & $33.7 \pm 25.4$ & $0.483 t$ \\
\hline Impact & $17.5 \pm 17.6$ & $18.2 \pm 18.0$ & $0.816 t$ \\
\hline Total & $21.1 \pm 13.1$ & $22.5 \pm 18.3$ & $0.837 t$ \\
\hline \multicolumn{4}{|c|}{ Hospital Anxiety and Depression Scale (HADS) } \\
\hline Anxiety & $4.5 \pm 8.8$ & $2.9 \pm 2.1$ & $0.624 t$ \\
\hline Depression & $1.6 \pm 2.4$ & $1.4 \pm 1.6$ & $0.830 t$ \\
\hline \multicolumn{4}{|l|}{ Pulmonary function testing } \\
\hline $\mathrm{FEV}_{1}(\mathrm{ml})$ & $2009 \pm 639$ & $2078 \pm 845$ & 0.816 \\
\hline $\mathrm{FEV}_{1} \%$ pred & $72.0 \pm 21.6$ & $77.3 \pm 28.0$ & 0.594 \\
\hline FVC (ml) & $3196 \pm 628$ & $3306 \pm 481$ & 0.621 \\
\hline FVC\%pred & $98.5 \pm 16.5$ & $105.8 \pm 12.2$ & 0.213 \\
\hline $\mathrm{FEV}_{1} / \mathrm{FVC}(\%)$ & $62.6 \pm 14.3$ & $62.6 \pm 22.1$ & 0.995 \\
\hline \multicolumn{4}{|l|}{ Incremental CPET } \\
\hline $\mathrm{VO}_{2}$ peak $(\mathrm{ml} / \mathrm{min} / \mathrm{kg})$ & $15.4 \pm 3.3$ & $14.4 \pm 3.9$ & 0.467 \\
\hline AT $(\mathrm{ml} / \mathrm{min} / \mathrm{kg})$ & $10.1 \pm 1.9$ & $9.7 \pm 2.1$ & 0.616 \\
\hline Peak work load (W) & $88.5 \pm 24.0$ & $82.8 \pm 27.5$ & 0.574 \\
\hline Breathing Reserve (L/min) & $41.69 \pm 21.52$ & $45.31 \pm 33.32$ & 0.511 \\
\hline
\end{tabular}

The data are expressed as the means \pm SD

Abbreviations: $A T$ anaerobic threshold, BMI body mass index, CPET cardiopulmonary exercise test, FEV 1 forced expiratory volume in $1 \mathrm{~s}, F V C$ forced vital capacity, $\mathrm{SpO}_{2}$ pluse oxygen saturation, VEGF-D vascular endothelial growth factor-D, $\mathrm{VO}_{2}$ peak peak oxygen consumption, 6MWD 6-min walking distance t The $P$ value was calculated with the use of the Wilcoxon rank-sum test

I The $P$ value was calculated with the use of Fisher's exact test 


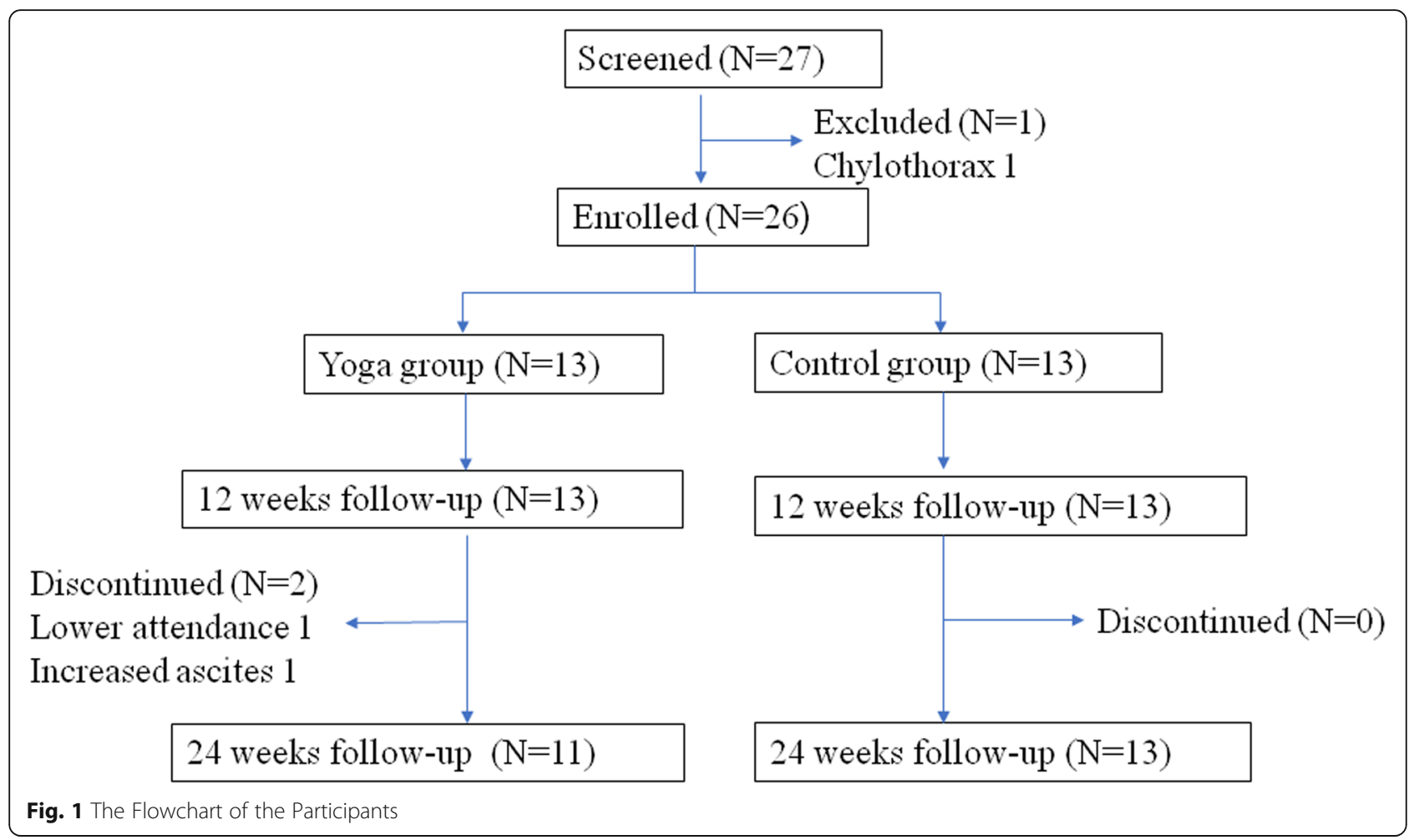

\section{Primary outcomes}

The difference in the 6MWD between the yoga and control groups was obvious over time. A significant difference was obtained at 24 weeks $(P=0.011)$ (Fig. 2). Within the groups, the increase in the 6MWD from baseline to 24 weeks was $18 \pm 49 \mathrm{~m}$ in the control group and $55 \pm 29 \mathrm{~m}$ in the yoga group. The post-intervention changes from baseline in the yoga group were significant $(P=0.04)$ (Fig. 2). $7 / 13$ from control group and $9 / 11$ from yoga group reached a $30-\mathrm{m}$ increase of 6MWD. The difference using that cut-off was not significant between groups $(P=0.211)$.

\section{Secondary outcomes}

Within each group, significant improvements emerged for $\mathrm{VO}_{2}$ peak (the yoga group $21.2 \pm 3.4 \mathrm{ml} / \mathrm{kg} / \mathrm{min}$ vs $15.4 \pm 3.5 \mathrm{ml} / \mathrm{kg} / \mathrm{min}, P=0.003$; the control group $18.5 \pm$ $5.5 \mathrm{ml} / \mathrm{kg} / \mathrm{min}$ vs $14.4 \pm 3.8 \mathrm{ml} / \mathrm{kg} / \mathrm{min}, P=0.016)$ and AT (the yoga group $13.1 \pm 2.7 \mathrm{ml} / \mathrm{kg} / \mathrm{min}$ vs $9.8 \pm 2.2 \mathrm{ml} /$ $\mathrm{kg} / \mathrm{min}, P=0.030$; the control group $11.3 \pm 2.4 \mathrm{ml} / \mathrm{kg} /$ min vs $9.7 \pm 2.1 \mathrm{ml} / \mathrm{kg} / \mathrm{min}, P=0.001)$. After a 24 -week intervention, the AT increased more in the yoga group than in the control group $(3.4 \pm 2.4 \mathrm{ml} / \mathrm{kg} / \mathrm{min}$ vs $1.6 \pm$ $1.4 \mathrm{ml} / \mathrm{kg} / \mathrm{min}, P=0.035$ ) (Fig. 2). However, in comparisons between the groups, no difference was found in the $\mathrm{VO}_{2}$ peak change $(5.8 \pm 2.2 \mathrm{ml} / \mathrm{kg} / \mathrm{min}$ vs $4.1 \pm 4.2 \mathrm{ml} / \mathrm{kg} /$ min, $P=0.224$ ) (Fig. 2). In addition, the peak work load in the incremental cardiopulmonary exercise test increased more in the yoga group than in the control group $(11.7 \pm 14.6 \mathrm{~W}$ vs $0.2 \pm 9.1 \mathrm{~W}, P=0.027)$ (Table 2 ) (Fig. 2).

There was no significant difference between the two groups with respect to the $\mathrm{FEV}_{1}$ or the FVC change from baseline to 24 weeks (Table 2).

There was no significant difference in anxiety and depression levels between the yoga group and the control group at baseline and 24 weeks, with adjustment for baseline levels of variables $(P=0.632,0.197)$.

There was no significant difference in the SGRQ slope between the two groups.

No pneumothorax, yoga-related injuries or other major adverse events were reported during the study.

\section{Discussion}

Our study showed that yoga for 24 weeks had beneficial effects in patients with LAM, especially for exercise tolerance. The main finding of this study was the significant improvement in the 6MWD and AT in the yoga group. No effects on $\mathrm{VO}_{2 \text { peak }}$, pulmonary function, dyspnea, anxiety and depression symptoms, or quality of life scores were observed. In addition, the yoga pattern was not difficult to achieve and maintain and was well tolerated by patients. The patients were adherent to the classes and able to safely participate after the 24-week program. 


\section{A}

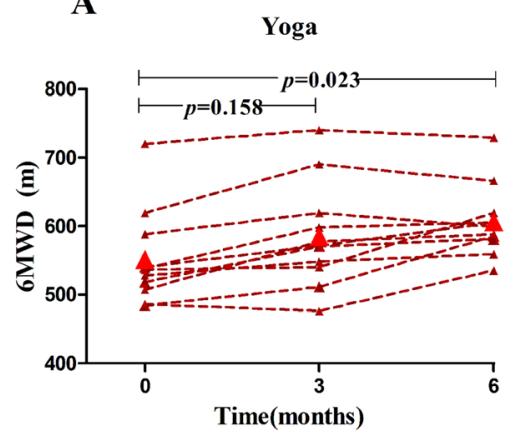

C

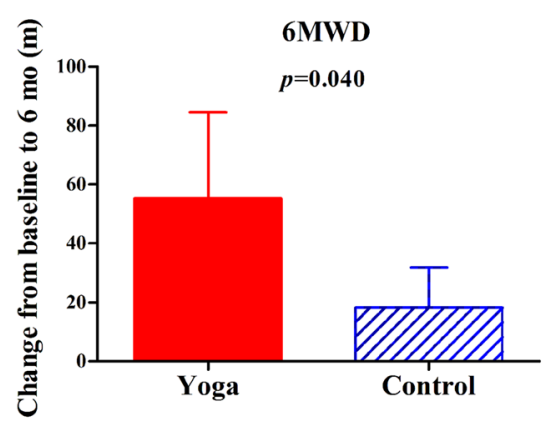

E

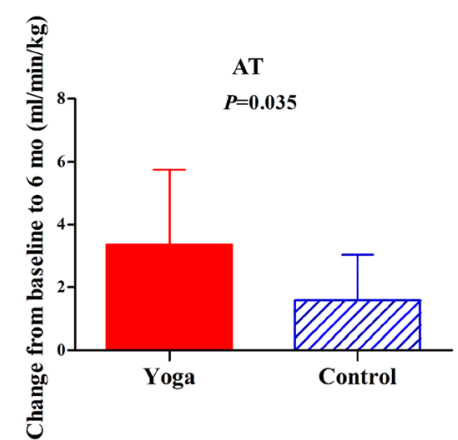

B

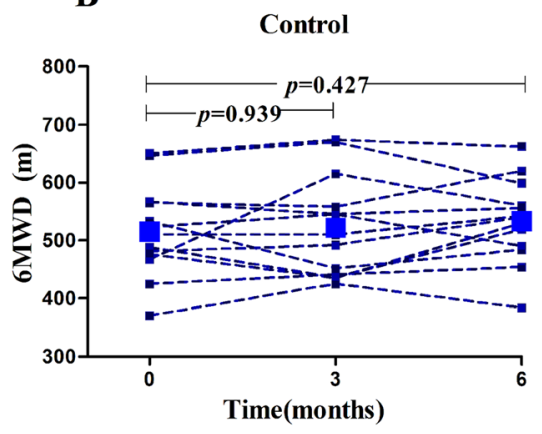

D

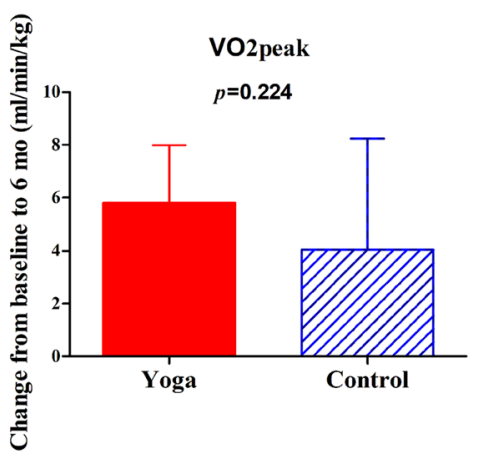

F

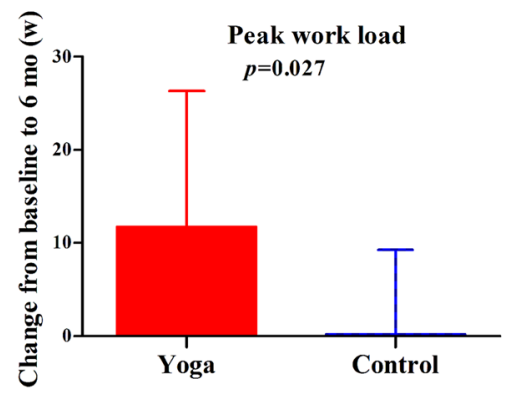

Fig. 2 Comparison of Six Minute Walking Test and Incremental Cardiopulmonary Exercise Test between Yoga and Control Groups. Six minute walking distance (6MWD) at baseline, 3 months and 6 months in yoga group (a) and control group (b). Changes of 6MWD (c), peak oxygen consumption $\left(\mathrm{VO}_{2}\right.$ peak) (d), anaerobic threshold (AT) (e) and peak work load (f) from baseline to 6 months after yoga exercise or control

Pulmonary rehabilitation is a standard practice that includes exercise training, education and behavior modification for patients with chronic lung diseases [8]. Such therapy can reduce dyspnea, increase exercise capacity, and improve quality of life. A controlled clinical trial consisting of 40 patients with LAM examined the effects of pulmonary rehabilitation [4]. The pulmonary rehabilitation group exhibited improved exercise endurance time, quality of life, 6MWD, and peak oxygen consumption. These results suggest that a pulmonary rehabilitation program should be employed in dyspneic patients with LAM. Despite compelling evidence for the benefit of pulmonary rehabilitation, only a very small percentage of eligible people attend a program. There are established barriers against enrollment and participation in center-based pulmonary rehabilitation programs related to referral practices, travel, transportation, disability and lack of program staffing $[9,10]$. Flexibility of site selection in yoga training makes it easy to adhere in the program.

Yoga originated in ancient India and may denote the union between the individual self and the transcendental self. It has been included as a component of exercises prescribed for many pulmonary rehabilitation programs. The body organs and systems are cleansed through asanas (postures) and pranayama (controlling the breath). Yoga 
Table 2 Effects of yoga on outcome variables

\begin{tabular}{|c|c|c|c|c|c|c|}
\hline \multirow[t]{2}{*}{ Variable } & \multicolumn{3}{|c|}{ Value at 6 months } & \multicolumn{3}{|c|}{ Change from baseline to 6 months } \\
\hline & Yoga $(\boldsymbol{N}=11)$ & Control $(\boldsymbol{N}=13)$ & $\boldsymbol{P}$ Value & Yoga $(N=11)$ & Control $(\boldsymbol{N}=13)$ & $\boldsymbol{P}$ Value \\
\hline Daily steps & $9305 \pm 3010$ & $8042 \pm 4431$ & 0.432 & $-912 \pm 2229$ & $-1346 \pm 1416$ & 0.585 \\
\hline 6MWD (m) & $606 \pm 53$ & $534 \pm 72$ & 0.011 & $55 \pm 29$ & $18 \pm 49$ & 0.040 \\
\hline $\mathrm{SpO}_{2}$ after exercise & $89 \pm 9$ & $89 \pm 9$ & 0.903 & $0.5 \pm 8.2$ & $-1.5 \pm 3.1$ & $0.058 t$ \\
\hline Borg score after exercise & $0.4 \pm 0.8$ & $0.6 \pm 0.8$ & $0.267 t$ & $-0.4 \pm 0.8$ & $-0.5 \pm 1.1$ & 0.737 \\
\hline VEGF-D, pg/ml & $2079 \pm 1149$ & $2913 \pm 2874$ & $0.977 t$ & $-183 \pm 660$ & $128 \pm 654$ & $0.505 t$ \\
\hline \multicolumn{7}{|c|}{ St. George Respiratory Questionnaire (SGRQ) } \\
\hline Symptoms & $9.6 \pm 10.5$ & $12.5 \pm 13.0$ & $0.681+$ & $-4.6 \pm 8.2$ & $-2.5 \pm 12.6$ & $0.416 t$ \\
\hline Activity & $26.5 \pm 13.5$ & $31.2 \pm 22.0$ & $0.485 t$ & $-2.4 \pm 11.6$ & $-2.5 \pm 7.8$ & $0.882+$ \\
\hline Impact & $8.8 \pm 5.2$ & $14.5 \pm 14.0$ & $0.640 t$ & $-3.0 \pm 10.2$ & $-3.7 \pm 10.1$ & $0.769+$ \\
\hline Total & $15.5 \pm 6.1$ & $19.3 \pm 15.0$ & $0.384 t$ & $-1.9 \pm 7.5$ & $-3.2 \pm 6.5$ & $0.682 t$ \\
\hline \multicolumn{7}{|c|}{ Hospital Anxiety and Depression Scale (HADS) } \\
\hline Anxiety & $0.9 \pm 1.4$ & $1.8 \pm 2.1$ & $0.283 t$ & $-0.8 \pm 1.5$ & $-1.1 \pm 1.8$ & $0.632 t$ \\
\hline Depression & $0.7 \pm 0.8$ & $0.8 \pm 1.2$ & $0.678 t$ & $-0.5 \pm 2.3$ & $-0.6 \pm 1.1$ & $0.197 \dagger$ \\
\hline \multicolumn{7}{|c|}{ Pulmonary function testing } \\
\hline FEV1 (ml) & $2062 \pm 612$ & $1974 \pm 770$ & 0.763 & $-29 \pm 182$ & $-104 \pm 143$ & $0.310+$ \\
\hline FEV1\%pred & $74.8 \pm 19.8$ & $74.3 \pm 25.2$ & 0.957 & $-0.8 \pm 6.6$ & $-3.0 \pm 5.5$ & $0.432 t$ \\
\hline FVC (ml) & $3252 \pm 606$ & $3225 \pm 457$ & 0.898 & $9 \pm 268$ & $-82 \pm 149$ & $0.581+$ \\
\hline FVC\%pred & $101.6 \pm 14.5$ & $105.5 \pm 14.0$ & 0.511 & $0.8 \pm 8.8$ & $-0.2 \pm 4.9$ & $0.681+$ \\
\hline FEV1/FVC & $62.8 \pm 12.7$ & $61.1 \pm 20.6$ & 0.803 & $-1.2 \pm 4.2$ & $5.3 \pm 23.8$ & $0.750 t$ \\
\hline \multicolumn{7}{|l|}{ Incremental CPET } \\
\hline $\mathrm{VO}_{2}$ Peak $(\mathrm{ml} / \mathrm{min} / \mathrm{kg})$ & $21.2 \pm 3.4$ & $18.5 \pm 5.5$ & 0.164 & $5.8 \pm 2.2$ & $4.1 \pm 4.2$ & 0.224 \\
\hline AT $(\mathrm{ml} / \mathrm{min} / \mathrm{kg})$ & $13.1 \pm 2.7$ & $11.3 \pm 2.4$ & 0.089 & $3.4 \pm 2.4$ & $1.6 \pm 1.4$ & 0.035 \\
\hline Peak work load (W) & $103.1 \pm 23.5$ & $82.9 \pm 27.6$ & 0.070 & $11.7 \pm 14.6$ & $0.2 \pm 9.1$ & 0.027 \\
\hline Breathing Reserve & $40.36 \pm 21.25$ & $46.46 \pm 29.47$ & 0.608 & $-3.27 \pm 8.91$ & $1.15 \pm 9.63$ & 0.186 \\
\hline
\end{tabular}

The data are expressed as the means \pm SD

$t$ The $p$ value was calculated with the use of the Wilcoxon rank-sum test Abbreviations: see Table 1

breathing or pranayama is beneficial for COPD. Previous research indicates that yoga is safe and feasible in participants with COPD [11]. Additionally, yoga participants with COPD have greater improvements in symptoms and functional performance.

In the present study, the 6MWD increase in the yoga group after 24 weeks was $55 \pm 29 \mathrm{~m}$. We observed a moderate increase in the $6 \mathrm{MWD}$ after yoga exercise; this increase is considered clinically relevant and superior to the variation obtained in a meta-analysis of COPD patients [5], which reported a mean difference of $39 \mathrm{~m}$ in the $6 \mathrm{MWD}$ after yoga training. We believe that yoga training has a positive effect on improving the exercise capacity of patients with LAM. When comparing groups using the generally accepted as clinically relevant cut-off for 6MWD of $30 \mathrm{~m}$, the difference found was not significant. However, both groups did have a relatively preserved walked distance in baseline test different from what is observed in other respiratory diseases. Considering that particularity a cut-off point of $60-\mathrm{m}$ increase might be better suited to compare groups which indeed showed a statistically significant difference favoring the intervention group (data not shown).

Though the peak oxygen consumption did not increase significantly in comparison to the control group, the improved AT might suggest an improvement in the aerobic capacity of the yoga group. These same results have been observed in COPD patients after practicing Hatha yoga $[12,13]$. The AT elevation was remarkably higher in the yoga group, suggesting a delayed onset of anaerobic metabolism and lactic acid accumulation after exercise training. When investigating the suggested mechanisms underlying the improvements observed in AT, peak work load and $6 \mathrm{MWD}$, it is reasonable to consider that different factors might be implied including desensitization to dyspnea and increased muscle 
strength. Also, it is not possible to completely exclude a motivation bias contributing to the greater performance in the intervention group. Another article regarding LAM rehabilitation described peripheral muscle adaptation resulting in better oxidative capacity as the main mechanism implied in the improvements they found [4]. Although that effect is classically associated with aerobic exercise it is possible to speculate that it could also happen with yoga if a significant elevation in heart rate and breath response were induced by the activity. However, we did not have enough evidence to support that hypothesis. The specific mechanisms implied need to be further studied.

$\mathrm{FEV}_{1}$ of both the control group and yoga group showed a significant reduction. The reduction rate of $\mathrm{FEV}_{1}$ in the yoga group was less than that in the control group; however, there were no significant differences between the two groups. A meta-analysis of COPD patients found that yoga training improved $\mathrm{FEV}_{1}$ [5].

Apart from relaxing tense muscles, yoga can also alleviate mental pressure [14]. Slow, relaxed breathing should also enhance well-being and reduce anxiety. A recent systematic review of the effects of yoga on anxiety found that the literature was poor quality and the effect on anxiety was equivocal [15]. Our study did not find significant improvements in depression and anxiety scales, but this might be partially explained by the fact that patients who joined this study did not have depression and anxiety exhibiting scores at baseline.

The Yoga exercise is quite safe. No pneumothorax or other serious adverse events related to yoga exercise were reported during the study.

There are some limitations to this study. First, we used 6MWD and incremental cardiopulmonary test as main measurements. Constant work rate exercise test should be more sensitive in reflecting the effects of intervention in daily life. Second, the study involved only 24 weeks of total duration and involved a small group of patients. Third, we did not specifically test participant comprehension of pranayama practice, but we did monitor that actual practice by a review at the next training. Fourth, we tried to enhance retention and adherence by having the subjects keep diaries and by reminding them to complete their daily practice through interactions with WeChat (a Chinese mobile social media application that provides instant messaging services to smart terminals) every day. Our data showed that on average, participants in the yoga group practiced $75 \mathrm{~min}$ per week at home, which could have reduced the impact of the intervention. Fifth, the impossibility of randomizing the intervention group, which was based on geographical issues, raised concern that the differences may existed in two groups although both groups seemed to be balanced in baseline characteristics, lung function and exercise capacity.

\section{Conclusions}

In conclusion, our findings demonstrated that yoga training may improve the functional exercise capacity in patients with LAM. Despite their frailty, patients diagnosed with LAM were able to safely perform yoga. Moreover, we suggest that yoga could be a useful adjunct pulmonary rehabilitation program for LAM patients. To help clarify the issue, further larger-scale trials with extended follow-up periods should be conducted to evaluate the long-term effects of yoga training in LAM patients.

\section{Supplementary information}

Supplementary information accompanies this paper at https://doi.org/10. 1186/s13023-020-1344-6.

Additional file 1 : Appendix. Online Supplementary information. Yoga exercise program used in this research for the participants with LAM.

\section{Abbreviations}

6MWD: 6-min walking distance; AT: Anaerobic threshold; BMI: Body mass index; COPD: Chronic obstructive pulmonary disease; CPET: Cardiopulmonary exercise test; $\mathrm{FEV}_{1}$ : Forced expiratory volume in $1 \mathrm{~s}$; FVC: Forced vital capacity; HADS: Hospital Anxiety and Depression Scale;

LAM: Lymphangioleiomyomatosis; NRDRS: National Rare Diseases Registry System of China; SGRQ: St. George Respiratory Questionnaire; $\mathrm{SpO}_{2}$ : Pluse oxygen saturation; VEGF-D: Vascular endothelial growth factor-D;

$\mathrm{VO}_{2}$ peak: Peak oxygen consumption

\section{Acknowledgments \\ The authors thank all LAM patients who participated in this study.}

\section{Authors' contributions}

Xu KF and Li X designed the study; Zu Y, Li Y, Xiang Y, Xiang Y and Chen L run the Yoga training; $X u$ W, Zhang $L$, Yang $Y$ collected data; Li $X, X u$ W, Liu W, Chen L and Xu KF analysed data; Li X, Xu W and Xu KF wrote the manuscript. All authors read and approved of the final version of the manuscript

\section{Funding}

The study was supported by the National Nature Science Foundation of China (81570061), the National Key Research and Development Program of China (2016YFC0901502, 2018YFC1313600), Chinese Academy of Medical Sciences (CAMS) Initiative for Innovative Medicine (2017-12 M-2-001), and LAM/TSC project of Peking Union Medical Foundation (2017-1).

\section{Availability of data and materials}

The dataset used in this research and analysis was available from the corresponding author

\section{Ethics approval and consent to participate}

The protocol was approved by the Peking Union Medical College Hospital ethical committee (ZS-1398), and all patients provided written informed consent before enrolment.

\section{Consent for publication}

Consent for publications were obtained from all participants.

\section{Competing interests}

The authors declare that they have no competing interests.

\section{Author details}

'Department of Rheumatology, Peking Union Medical College Hospital, Chinese Academy of Medical Sciences, Peking Union Medical College, Beijing 100730, China. ${ }^{2}$ Department of Pulmonary and Critical Care Medicine, Peking Union Medical College Hospital, Chinese Academy of Medical Sciences, 
Peking Union Medical College, Beijing 100730, China. ${ }^{3}$ Department of Rehabilitation, Peking Union Medical College Hospital, Chinese Academy of Medical Sciences, Peking Union Medical College, Beijing 100730, China. ${ }^{4}$ Beijing Hexin Sunshine Sports Culture Co. Ltd, Beijing, China. ${ }^{5}$ LAM Yoga Project, Beijing, China. ${ }^{6}$ Department of International Medical Service, Peking Union Medical College Hospital, Chinese Academy of Medical Sciences, Peking Union Medical College, Beijing 100730, China. ${ }^{7}$ Rare Diseases Research Center, Chinese Academy of Medical Sciences, Beijing, China.

Received: 19 August 2019 Accepted: 2 March 2020

Published online: 16 March 2020

\section{References}

1. McCormack FX, Travis WD, Colby TV, Henske EP, Moss J.

Lymphangioleiomyomatosis: calling it what it is: a low-grade, destructive, metastasizing neoplasm. Am J Respir Crit Care Med. 2012;186(12):1210-2.

2. McCormack FX, Inoue $Y$, Moss J, Singer LG, Strange C, Nakata $K$, et al. Efficacy and safety of sirolimus in lymphangioleiomyomatosis. N Engl J Med. 2011:364(17):1595-606.

3. Baldi BG, Albuquerque AL, Pimenta SP, Salge JM, Kairalla RA, Carvalho CR. Exercise performance and dynamic hyperinflation in lymphangioleiomyomatosis. Am J Respir Crit Care Med. 2012;186(4):341-8.

4. Araujo MS, Baldi BG, Freitas CS, Albuquerque AL, Marques da Silva CC, Kairalla RA, et al. Pulmonary rehabilitation in lymphangioleiomyomatosis: a controlled clinical trial. Eur Respir J. 2016;47(5):1452-60.

5. Liu XC, Pan L, Hu Q, Dong WP, Yan JH, Dong L. Effects of yoga training in patients with chronic obstructive pulmonary disease: a systematic review and meta-analysis. J Thoracic Dis. 2014;6(6):795-802.

6. Gupta N, Finlay GA, Kotloff RM, Strange C, Wilson KC, Young LR, et al. Lymphangioleiomyomatosis diagnosis and management: high-resolution chest computed tomography, Transbronchial lung biopsy, and pleural disease management. An official American Thoracic Society/Japanese respiratory society clinical practice guideline. Am J Respir Crit Care Med. 2017;196(10):1337-48.

7. Brooks D, Solway S, Gibbons WJ. ATS statement on six-minute walk test. Am J Respir Crit Care Med. 2003;167(9):1287.

8. Spruit MA, Singh SJ, Garvey C, ZuWallack R, Nici L, Rochester C, et al. An official American Thoracic Society/European Respiratory Society statement: key concepts and advances in pulmonary rehabilitation. Am J Respir Crit Care Med. 2013;188(8):e13-64.

9. Keating A, Lee A, Holland AE. What prevents people with chronic obstructive pulmonary disease from attending pulmonary rehabilitation? A systematic review. Chron Respir Dis. 2011;8(2):89-99.

10. Cox NS, Oliveira CC, Lahham A, Holland AE. Pulmonary rehabilitation referral and participation are commonly influenced by environment, knowledge, and beliefs about consequences: a systematic review using the theoretical domains framework. J Physiother. 2017;63(2):84-93.

11. Donesky-Cuenco D, Nguyen HQ, Paul S, Carrieri-Kohlman V. Yoga therapy decreases dyspnea-related distress and improves functional performance in people with chronic obstructive pulmonary disease: a pilot study. J Altern Complement Med. 2009;15(3):225-34.

12. Ranjita R, Hankey A, Nagendra HR, Mohanty S. Yoga-based pulmonary rehabilitation for the management of dyspnea in coal miners with chronic obstructive pulmonary disease: a randomized controlled trial. J Ayurveda Integr Med. 2016;7(3):158-66.

13. Papp $M E$, Wandell $P E$, Lindfors $P$, Nygren-Bonnier $M$. Effects of yogic exercises on functional capacity, lung function and quality of life in participants with obstructive pulmonary disease: a randomized controlled study. Eur J Phys Rehabil Med. 2017;53(3):447-61.

14. Sharma VK, Das S, Mondal S, Goswampi U, Gandhi A. Effect of Sahaj yoga on depressive disorders. Indian J Physiol Pharmacol. 2005;49(4):462-8.

15. Kirkwood G, Rampes H, Tuffrey V, Richardson J, Pilkington K. Yoga for anxiety: a systematic review of the research evidence. Br J Sports Med. 2005; 39(12):884-91 discussion 91.

\section{Publisher's Note}

Springer Nature remains neutral with regard to jurisdictional claims in published maps and institutional affiliations.

\section{Ready to submit your research? Choose BMC and benefit from:}

- fast, convenient online submission

- thorough peer review by experienced researchers in your field

- rapid publication on acceptance

- support for research data, including large and complex data types

- gold Open Access which fosters wider collaboration and increased citations

- maximum visibility for your research: over $100 \mathrm{M}$ website views per year

At BMC, research is always in progress.

Learn more biomedcentral.com/submissions 